\title{
Editorial about PROOFS 2019
}

\author{
Karine Heydemann ${ }^{1}$ - Letitia $\mathrm{Li}^{2}$
}

Published online: 5 August 2020

(c) Springer-Verlag GmbH Germany, part of Springer Nature 2020

The goal of the PROOFS workshop is to promote methodologies that provably improve the security of embedded systems, especially those which contain cryptographic algorithms. More specifically, the PROOFS workshop seeks contributions in both theory and practice of methods and tools applied to the security of embedded systems. Examples include (semi-)formal methods, simulation-based leakage evaluation and security checks, protocol verification techniques, test and verification of secure embedded systems (software and hardware), and provable security for physical attacks.

This year's PROOFS workshop was held on August 24, 2019, in Atlanta, Georgia, USA. Georgia is one of the hubs of cybersecurity around the world, home to industrial and government centers of research as well as academic institutions. The $120+$ cybersecurity companies in the state generate nearly $25 \%$ of the security market around the world. The workshop was held one day before the colocated conference CHES (Cryptographic Hardware and Embedded Systems), the flagship IACR conference on embedded systems security.

PROOFS 2019 took place at a time where the world has begun to recognize the importance of developing security countermeasures against the rising tide of cyberattacks, including hardware-based ones on the increasing prevalent devices within the Internet of things. 2019 saw hacks on smartphones, massive data breaches resulting in disclosure of personal data, and costly ransomware attacks.

The presentations of PROOFS addressed these issues, starting with a keynote by Professor Ryan Gerdes of Virginia Tech, who discussed the attacks on transportation systems as well as the hardware-based geofencing to protect sensitive sites, such as airports.

The second session started with Professor Jakub Szefer's talk on cache security, a subject of increasing importance demonstrated by the famous Spectre and Meltdown attacks,

Karine Heydemann

karine.heydemann@lip6.fr

Sorbonne University, CNRS, LIP6, 75005 Paris, France

2 FAST Labs, BAE Systems, Burlington, MA 01803, USA regarding modeling timing channels and a new security benchmark suite to quantitatively assess caches.

The rest of the program included the presentations of five contributed papers, whose authors were invited to submit revised papers to this special issue of Journal of Cryptographic Engineering on PROOFS.

The PROOFS workshop organizers are indebted to the program committee for its hard work in reading, evaluating, and commenting the submissions. We would like to sincerely acknowledge the work of the program committee of PROOFS 2019, which was made up of:

- Ludovic Apvrille, Telecom ParisTech

- Manuel Barbosa, HASLab - INESC TEC and FCUP

- Shivam Bhasin, Temasek Labs@NTU

- Begül Bilgin, Rambus Cryptography Research and KU Leuven

- Lukasz Chmielewski, Riscure

- Damien Couroussé, CEA

- Giorgio Di Natale, LIRMM

- Fatemeh Ganji, Florida Institute for Cybersecurity Research

- Naofumi Homma, Tohoku University

- Ulrich Kühne, Telecom ParisTech

- Kerstin Lemke-Rust, Bonn-Rhein-Sieg University of Applied Sciences

- Quentin Meunier, LIP6

- Debdeep Mukhopadhyay, IIT Kharagpur

- Renaud Pacalet, Telecom ParisTech

- Stjepan Picek, Delft University of Technology

- Ilia Polian, University of Stuttgart

- Erik Poll, Radboud University

- Francesco Regazzoni, ALaRI - USI (Switzerland)

- Gilles Van Assche, STMicroelectronics

They were assisted by 2 subreviewers:

- Jean-Luc Danger, Telecom ParisTech

- Lauren De Meyer, KU Leuven 
Finally, I also thank all the participants of the workshop, for whom we hope PROOFS 2019 was a source of inspiration and collaboration for future projects in the security of embedded systems.
Karine Heydemann, LIP6

Letitia Li, BAE Systems Co-program chairs of PROOFS 2019.

Publisher's Note Springer Nature remains neutral with regard to jurisdictional claims in published maps and institutional affiliations. 